\title{
A New Method for Analyzing Electron Backscatter Diffraction (EBSD) Data for Texture using Inverse Pole Figures
}

\author{
C. T. Chou*, P. Rolland*, K. G. Dicks* \\ * Oxford Instruments Analytical, Halifax Road, High Wycombe HP12 3SE UK
}

Crystallographic texture or preferred orientation in a plate or thin film polycrystalline sample is commonly displayed as $\{\mathrm{h} \mathrm{k} \mathrm{l}\}\langle\mathrm{u} \mathrm{v} \mathrm{w}\rangle$. $\{\mathrm{h} \mathrm{k} \mathrm{l}\}$ represents the crystal plane normal that is parallel to the sample Normal Direction (ND) and $\langle\mathrm{u} \mathrm{v} \mathrm{w}\rangle$ represents the crystal direction that is parallel to the sample longitudinal or Rolling Direction (RD). Traditional methods for texture determination in polycrystalline materials involve the inspection of Pole Figures (PFs) or Euler plots/ODF [1],[2],[3]. An alternative approach has been developed for EBSD data, which uses two Inverse Pole Figures (IPFs) to identify and quantify texture(s) present. The new method is very straightforward, interactive, and requires no expert knowledge or interpretation. The new approach is made possible because EBSD provides the orientation at every pixel in a Crystal Orientation Map (COM), directly as $\{h k l\}\langle u v w>$.

\section{Pole figure (PF) method:}

PFs are stereographic projections for chosen crystal directions (e.g. <111> directions). Usually inspection of a number of PFs for different crystal directions (e.g. 100, 110, 111, and others) will be required in order to derive the sample textures. Interpretation often requires a degree of expertise. Further, it can be difficult to resolve superimposed patterns of data relating to different texture components.

\section{Euler space method:}

A crystal orientation can also be represented by three consecutive rotations relative to the sample (Euler angles $\phi 1, \Phi$, and $\phi 2$ ). The results can be displayed graphically in '3D' Euler space, and texture will be shown by the formation of clusters of points. The textures of $\{\mathrm{hkl}\}<\mathrm{uvw}>$ are then obtained, by comparing the values of the Euler angles with calculations. Using Euler space to visualise textures is not straightforward, and significant knowledge is required to determine $\{h k l\}<u v w>$ from the Euler angles distribution.

\section{Inverse pole figure method:}

Considering X Ray Diffraction (XRD) data, IPFs can be used to identify fiber texture, but not as an universal means for texture analysis [1],[2]. In XRD the use of IPFs is limited because the correlation of $\{\mathrm{hkl}\}$ and $\langle\mathrm{uvw}>$ cannot be determined. However, in EBSD, the $\{\mathrm{hkl}\}\langle\mathrm{uvw}>$ couple is obtained for every measurement which makes texture analysis using IPFs possible. An acceptance angle to include similar directions can be directly visualized in the IPF. Thus, selecting a plane $\{\mathrm{hkl}\}$ with a chosen tolerance $\left(\mathrm{n}^{\circ}\right)$ allows the generation of a new IPF showing the $\langle\mathrm{uvw}\rangle$ distribution of the selected $\{h \mathrm{hl}\}$. The distribution of the selected $\{\mathrm{hkl}\}$ range: $\{\mathrm{hkl}\}\langle\mathrm{uvw}\rangle$ i.e. texture, is visualized directly using Miller indices, without conversion. It takes into account symmetries and is interactive with the Crystal Orientation Map (COM). 


\section{Using two inverse pole figures to display texture from EBSD data}

A ND IPF directly plots a plane $\{h k l\}$ as a single unique point. Similarly, the RD IPF plots the direction $\langle u v w\rangle$. Thus many orientation measurements can be shown in an IPF, displayed as either a discrete plot (Fig. 2) where each measurement is represented by a point, or as density plots (fig. 2'). Thus the distribution of $\{\mathrm{hkl}\}$ or $\langle\mathrm{uvw}\rangle$ is visualized. A EBSD IPF colorkey is used for the COM (Fig. 1). A dominant $\{\mathrm{hkl}\}$ distribution will be seen as a dominant color in the ND COM. Using EBSD data, all the planes $\{$ hkl $\}$ of the same direction, within a tolerance $\left(n^{\circ}\right)$, can be extracted graphically from the ND IPF, or from the ND COM. Then the distribution of all directions <uvw> normal to the selected $\{\mathrm{hkl}\}$ (Fig 3) can be seen in a single plot (Fig 4) in the RD IPF. The most common $\langle\mathrm{uvw}\rangle$ is visible, as well as the angle over which it spreads. This can be selected and gives the selected texture frequency and location directly as $\{\mathrm{hkl}\}\langle\mathrm{uvw}\rangle$. The $\langle\mathrm{uvw}\rangle$ shared with another $\{h k l\}$ can be discriminated as in the case $\{111\}<1-10>$ and $\{110\}<1-10>$.

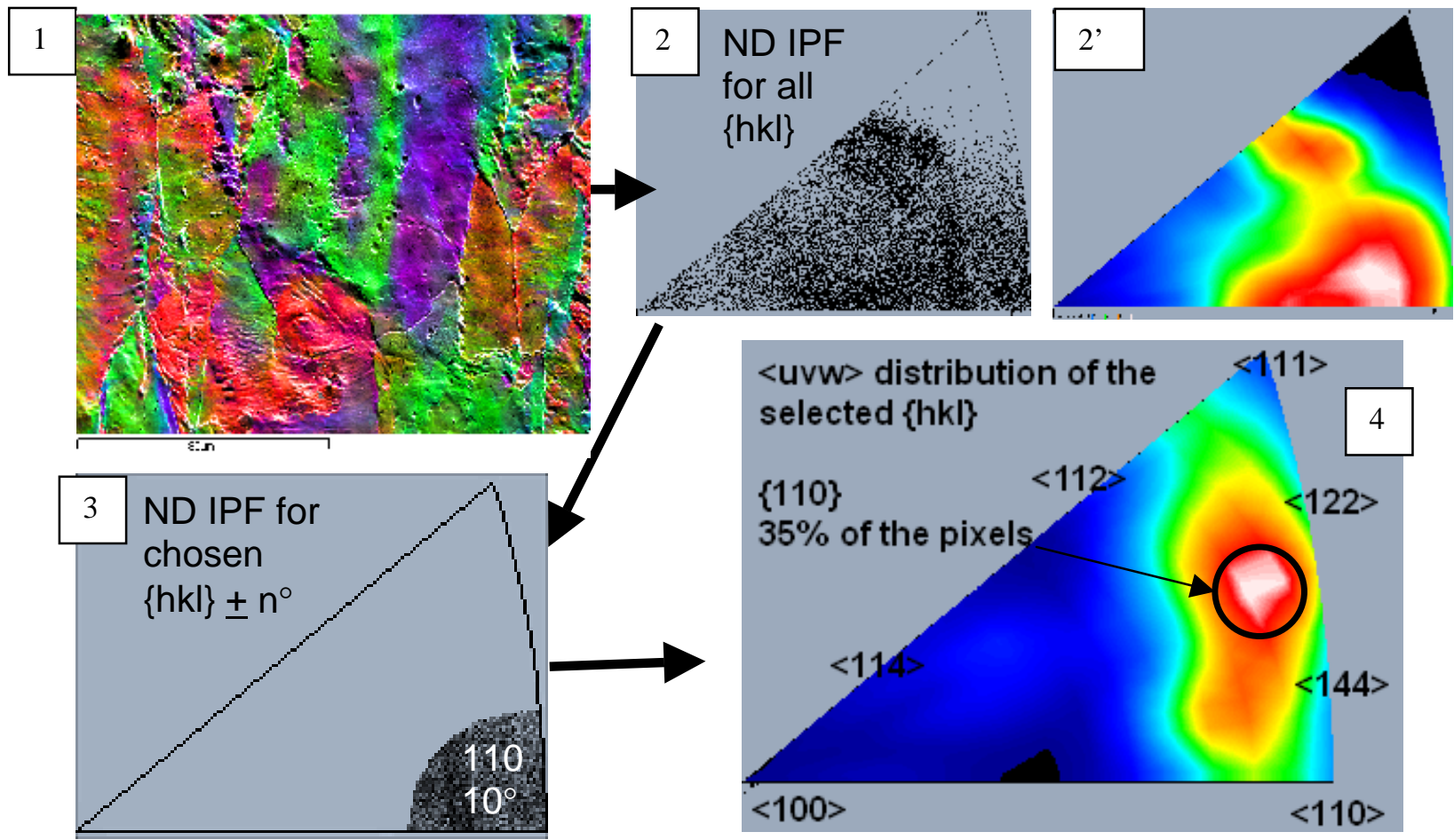

Above: procedure for extracting all $\{110\}$ with $10^{\circ}$ tolerance (fig 3) from the COM (fig. 1). The distribution of all directions <uvw> perpendicular to $\{110\} \pm 10^{\circ}$ is shown in fig. 4 . The circle indicates that $35 \%$ of measurements fulfill that criterion. In this example the concentration of planes was in fact closer to $\left\{\begin{array}{lll}1 & 9 & 12\end{array}\right\}$. If $\left\{\begin{array}{lll}1 & 9 & 12\end{array}\right\}$ with $7^{\circ}$ tolerance is selected, $45 \%$ of the pixels fulfill this criterion. This shows the flexibility, selectivity, accuracy and interactivity of the approach.

[1] V. Randle and O. Engler: Introduction to texture analysis, macrotexture, microtexture and orientation mapping, Gordon and Breach Science Publishers, 2000.

ISBN 9056992244.

[2] U.F. Kocks, C.N. Tomé, and H. R. Wenk: Texture and Anisotropy, Cambridge University Press, 1998. ISBN 0521465168.

[3] H. J. Bunge: Texture analysis in materials science, Butterworth \& Co, 1982. ISBN 0408106425. 